\title{
Semispinalis Thoracis
}

National Cancer Institute

\section{Source}

National Cancer Institute. Semispinalis Thoracis. NCI Thesaurus. Code C53006.

One of the transversospinales group of muscles that arises from C6-C7 cervical and T1-

T4 spinous processes, and inserts into the transverse processes of the T6-T10

vertebrae; it functions to extend the spine and rotate it toward the contralateral side. 